\title{
Ligation-Independent Mechanism of Multiplex Ligation-Dependent Probe Amplification
}

\author{
Naoki UNO ${ }^{\dagger}$ and Katsunori YANAGIHARA \\ Department of Laboratory Medicine, Nagasaki University Graduate School of Biomedical Sciences, 1-7-1 \\ Sakamoto, Nagasaki 852-8501, Japan
}

\begin{abstract}
Multiplex ligation-dependent probe amplification (MLPA) is a widely used technique for detecting genomic structural variants. The technique is based on hybridization and ligation, followed by amplification of the ligation products. Therefore, ligation is considered a fundamental process that determines the feasibility and fidelity of MLPA. However, despite the widespread use of this technique, its reaction mechanism has not been fully analyzed. Herein, we describe a ligation-independent pathway for MLPA and introduce a ligation-independent probe amplification system that can be used to obtain amplified products without the hybridization and ligation processes. Fragment analysis revealed that the ligation-independent pathway is functional and that the capacity to discriminate single nucleotides with MLPA does not depend on ligation. These findings indicate that the feasibility and fidelity of MLPA do not rely on ligation.
\end{abstract}

Keywords MLPA, ligation, fragment analysis, helicase-dependent amplification

(Received May 13, 2014; Accepted June 24, 2014; Published August 10, 2014)

\section{Introduction}

Multiplex ligation-dependent probe amplification (MLPA) is a polymerase chain reaction (PCR)-based technique initially developed in 2002. ${ }^{1}$ Since then, it has rapidly become a popular method for detecting copy number variations (CNVs). Although many techniques for the detection of structural variants have been developed, ${ }^{2-4}$ MLPA is popular because of its ease of use. ${ }^{5}$ Its usefulness has been validated by many reports, and various MLPA-based applications have been developed for both diagnostic and research use. ${ }^{6-9}$ Although MLPA has proved a reliable tool for copy number analysis, it has been poorly validated for single-nucleotide analysis. MLPA is mainly used for copy number analysis, but it can also be used for the detection of point mutations. ${ }^{9,10}$ The latter application is based on the principle that MLPA is specific enough to distinguish a single nucleotide in a template owing to the high fidelity of ligation. However, in reality, MLPA has seldom been used for that purpose. Although MLPA offers great specificity for detecting CNVs, its capacity to detect single-nucleotide alterations is unclear.

According to its principle, MLPA uses right and left probes that hybridize to a denatured template (Fig. 1). Overnight incubation allows both probes to hybridize to their targets. Subsequent ligation links both probes as the left and right probe are adjacent to each other and the $5^{\prime}$ end of the right probe is phosphorylated. Once ligation occurs, the ligation products can serve as templates for subsequent PCR. A polymerase synthesizes new strands complementary to the ligation products from the reverse primer because the $3^{\prime}$ end of the right probe is

$\dagger$ To whom correspondence should be addressed.

E-mail: nuno@nagasaki-u.ac.jp complementary to the reverse primer. The forward primer then anneals to the $3^{\prime}$ end of the newly synthesized strands because the sequence of the $5^{\prime}$ end of the left probe is identical to that of the forward primer. Thus, after the ligation of the right and left probes, PCR readily amplifies the ligation products. Ligation is therefore considered a critical step in determining the feasibility and specificity of MLPA, because in principle, no product is amplified without ligation in MLPA. For the same reason, MLPA is expected to be as specific as the process of ligation. However, the reaction mechanism underlying MLPA has not been analyzed in detail. In particular, the role of ligation in single-base change discrimination remains to be elucidated.

In the present study, we questioned the importance of ligation in MLPA and investigated a ligation-independent mechanism for MLPA. Fragment analysis revealed that the ligation-independent pathway is functional and that ligation is insufficient for discriminating single-nucleotide changes in MLPA.

\section{Experimental}

\section{Oligonucleotides}

All oligonucleotide templates and left probes were synthesized and purified with polyacrylamide electrophoresis by Sigma Genosys (Hokkaido, Japan). For fragment analysis, the $5^{\prime}$ end of the left probe was labeled with FAM. Right probes were synthesized and purified with either polyacrylamide electrophoresis or a reverse-phase cartridge by Sigma-Genosys. For the ligation reaction, the $5^{\prime}$ end of the right probe was phosphorylated. Forward and reverse primers were synthesized and purified with a reverse-phase cartridge by Sigma Genosys and desalted by Applied Biosystems (Foster City, CA), respectively. For fragment analysis, the $5^{\prime}$ end of the reverse 


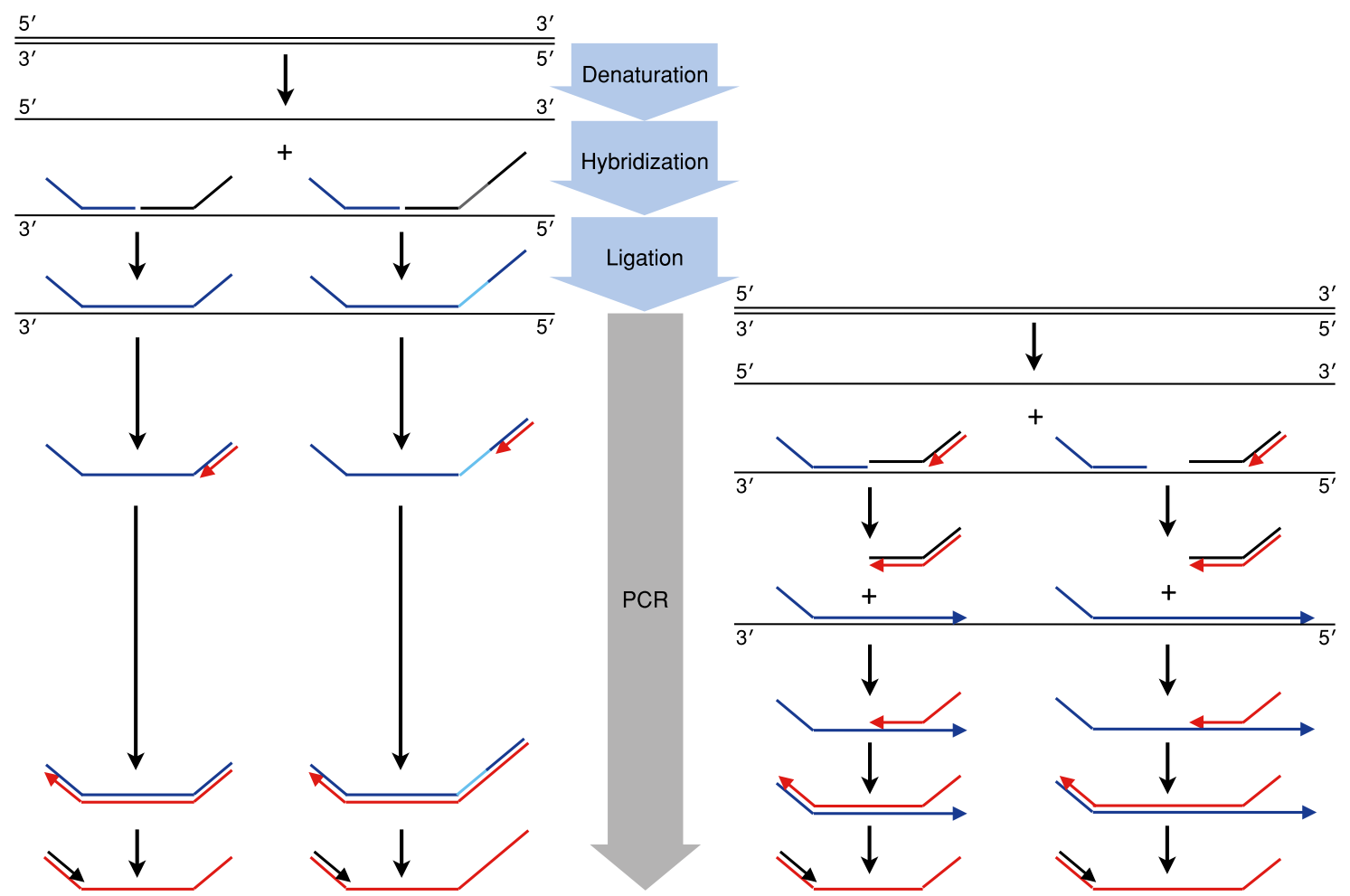

Fig. 1 Schematic representation of multiplex ligation-dependent probe amplification (MLPA) and ligation-independent probe amplification.

primer was labeled with PET by Applied Biosystems. Sequences of all oligonucleotides are shown in Table S1 (Supporting Information).

Pilot experiment testing the feasibility of ligation-independent probe amplification

Reaction mixtures containing $10 \mathrm{nM}$ left probe, $10 \mathrm{nM}$ right probe, $10 \mathrm{nM}$ template, $10 \mathrm{nM}$ reverse primer, $1 \times$ Annealing Buffer II (10 mM KCl, $20 \mathrm{mM}$ Tris-HCl, pH 8.8) (BioHelix, Beverly, MA), $50 \mathrm{mM} \mathrm{NaCl}, 3 \mathrm{mM} \mathrm{MgSO}$, $3 \mathrm{mM}$ dATP (Takara Bio, Shiga, Japan), $0.2 \mathrm{mM}$ deoxyribonucleotide triphosphates (dNTPs), $12.5 \mathrm{ng} / \mu \mathrm{L}$ Thermostable Helicase (BioHelix), and 0.1 U/ $/ \mathrm{L}$ Bst Polymerase Large Fragment (New England Biolabs, Ipswich, MA) were incubated at $65^{\circ} \mathrm{C}$ for $15 \mathrm{~min}$.

\section{Fragment analysis}

Aliquots $(3 \mu \mathrm{L})$ were removed from the reaction mixtures and immediately mixed with $7.5 \mu \mathrm{L}$ of stop solution containing $7 \mu \mathrm{L}$ of Hi-Di Formamide (Applied Biosystems) and $0.5 \mu \mathrm{L}$ of GeneScan 600 LIZ Size Standard v 2.0 (Applied Biosystems) at $65^{\circ} \mathrm{C}$. Samples were then heated at $95^{\circ} \mathrm{C}$ for $3 \mathrm{~min}$ and chilled on ice until electrophoresis. Samples were separated with capillary electrophoresis through POP-7 polymer (Applied Biosystems) on a 3130 Genetic Analyzer (Applied Biosystems). Spectral calibration was performed using DS-33 Matrix Standards (Applied Biosystems). Data were analyzed using a Peak Scanner (Applied Biosystems).
Ligation-independent probe amplification using helicase-dependent amplification (HDA)

Reaction mixtures containing $4 \mathrm{nM}$ left probe, $4 \mathrm{nM}$ right probe, $4 \mathrm{nM}$ template, $24 \mathrm{nM}$ forward and reverse primer, $1 \times$ Annealing Buffer II $(10 \mathrm{mM} \mathrm{KCl}, 20 \mathrm{mM}$ Tris-HCl, $\mathrm{pH} 8.8)$ (BioHelix), $80 \mathrm{mM} \mathrm{NaCl}, 5 \mathrm{mM} \mathrm{MgSO} 4,3 \mathrm{mM}$ dATP (Takara Bio), $0.2 \mathrm{mM}$ dNTPs, $12.5 \mathrm{ng} / \mu \mathrm{L}$ Thermostable Helicase (BioHelix), and 0.1 U/ $\mu \mathrm{L}$ Bst Polymerase Large Fragment (New England Biolabs) were incubated at $65^{\circ} \mathrm{C}$ for $15 \mathrm{~min}$. Aliquots $(3 \mu \mathrm{L})$ were removed at the indicated time, and the reactions were terminated as described for fragment analysis

\section{Ligation-independent probe amplification using PCR}

Reaction mixtures containing $4 \mathrm{nM}$ left probe, $4 \mathrm{nM}$ right probe, $4 \mathrm{nM}$ template, $24 \mathrm{nM}$ forward and reverse primer, $1 \times$ PCR Buffer (Takara Bio), $0.2 \mathrm{mM}$ dNTPs, and $0.05 \mathrm{U} / \mu \mathrm{L}$ Taq polymerase (Takara Bio) were prepared on ice, and PCR was performed under the following conditions: $95^{\circ} \mathrm{C}$ for $30 \mathrm{~s}, 60^{\circ} \mathrm{C}$ for $30 \mathrm{~s}$, and $72^{\circ} \mathrm{C}$ for $1 \mathrm{~min}$. Aliquots $(3 \mu \mathrm{L})$ were removed at the indicated cycle, and the reactions were terminated as described for fragment analysis.

\section{MLPA reaction}

Ligations were performed in reaction mixtures containing $10 \mathrm{nM}$ left probe, $10 \mathrm{nM}$ phosphorylated right probe, $10 \mathrm{nM}$ template, $1 \times$ Taq DNA ligase reaction buffer (New England Biolabs), and $1 \mathrm{U} / \mu \mathrm{L}$ Taq DNA ligase (New England Biolabs). Reaction mixtures were incubated at $65^{\circ} \mathrm{C}$ for $15 \mathrm{~min}$ and immediately placed on ice or cooled to $4^{\circ} \mathrm{C}$. PCR reaction mixtures containing 0.4 volume of the ligation mixtures, $24 \mathrm{nM}$ forward and reverse primer, $0.2 \mathrm{mM}$ dNTPs, $1 \times$ PCR buffer 


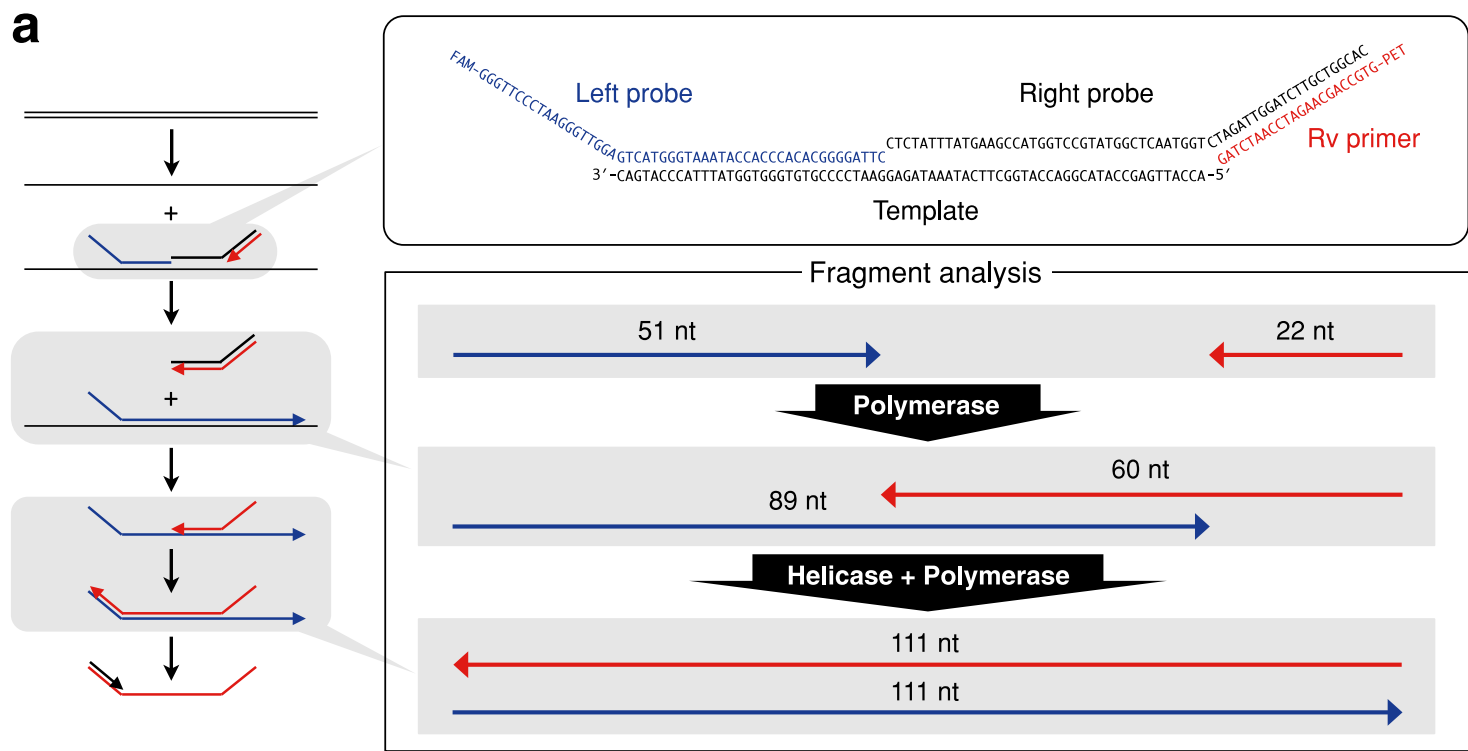

b

Water
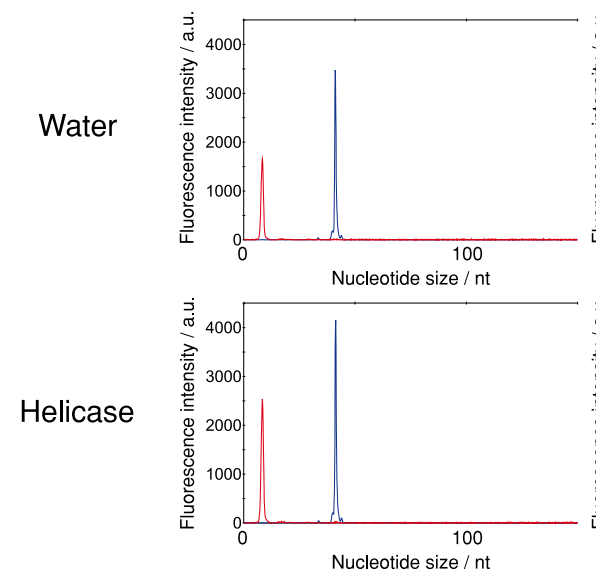

Polymerase
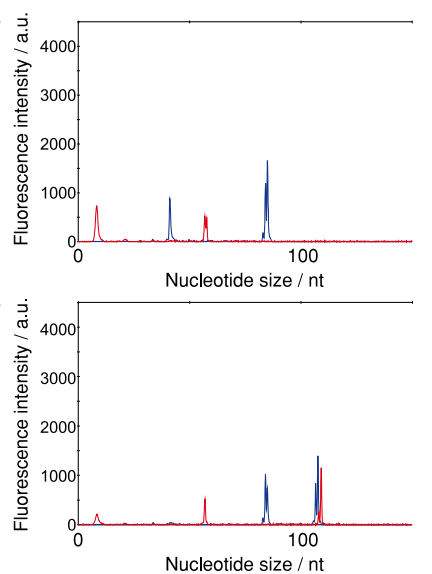

Fig. 2 Pilot experiment testing the feasibility of ligation-independent probe amplification. (a) The left probe and reverse primer are fluorescently labeled with FAM (blue) and PET (red), respectively, so that each process of the reaction can be monitored with fragment analysis. Arrowheads indicate the direction of $5^{\prime} \rightarrow 3^{\prime}$ DNA synthesis. (b) A 111-nt fragment labeled with PET is generated when both the polymerase and the helicase are present. Fragments labeled with FAM and PET are shown in blue and red, respectively.

(Takara Bio), and $0.05 \mathrm{U} / \mu \mathrm{L}$ Taq polymerase (Takara Bio) were prepared on ice, and PCR was carried out under the conditions of $95^{\circ} \mathrm{C}$ for $30 \mathrm{~s}, 60^{\circ} \mathrm{C}$ for $30 \mathrm{~s}$, and $72^{\circ} \mathrm{C}$ for $1 \mathrm{~min}$. Aliquots $(3 \mu \mathrm{L})$ were removed before the reaction and during the $72^{\circ} \mathrm{C}$ step of the indicated cycle. The reactions were terminated as described for fragment analysis.

\section{Results}

\section{Ligation-independent pathway of MLPA}

We designed a ligation-independent pathway for MLPA through which the same products could be amplified without the hybridization and ligation processes (Fig. 1). Double-stranded DNA is denatured using this pathway, and subsequently, the reverse primer anneals to the $3^{\prime}$ end of the right probe, whereas the left probe anneals to the denatured template. DNA polymerase then synthesizes new strands from the reverse primer and the left probe. After a second denaturation, both newly synthesized strands anneal, and DNA polymerase further extends the reverse primer to the $5^{\prime}$ end of the left probe. In MLPA, DNA polymerase synthesizes the same strand from the reverse primer using the ligation product as the template and amplifies it using both the forward and the reverse primers. Therefore, MLPA is theoretically possible without the hybridization and ligation processes.

\section{Ligation-independent probe amplification is feasible}

We first conducted a pilot experiment to test the feasibility of the reaction we designed. To simplify the reaction, we used a single probe pair without a gap, an oligonucleotide template, and a reverse primer (Fig. 2a). The probes and primer were designed according to the protocol used for designing synthetic MLPA probes (MRC-Holland, Amsterdam, The Netherlands). The $5^{\prime}$ end of the right probe was not phosphorylated because ligation was not performed. To monitor the reaction in detail, we performed fragment analysis by fluorescently labeling the left probe and the reverse primer with FAM and PET, respectively 


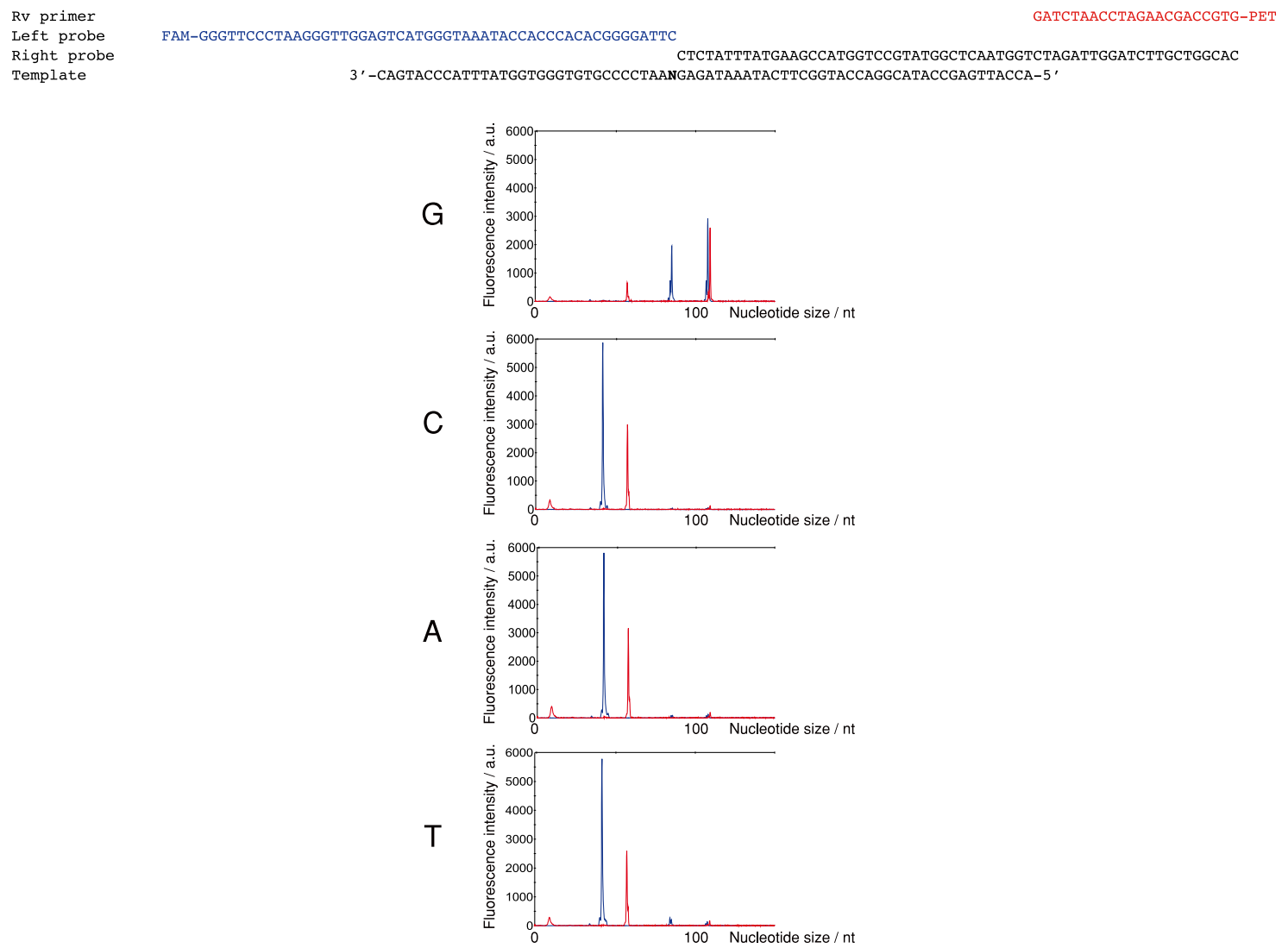

Fig. 3 A mismatch between the $3^{\prime}$ end of the left probe and the template inhibits the extension of the left probe by the polymerase. Reaction conditions were the same as those illustrated in Fig. 2, except that templates differing by a single nucleotide (bold) were used. The discriminating base is shown on the left side of each electropherogram. Fragments labeled with FAM and PET are shown in blue and red, respectively.

(Fig. 2a). We used DNA helicase rather than heat denaturation to unwind the double-stranded DNA to preserve isothermal DNA amplification. ${ }^{11,12}$

Two newly synthesized fragments were observed in the presence of the polymerase and absence of the helicase (Fig. 2b): an 89-nt fragment labeled with FAM and a 60-nt fragment labeled with PET. Therefore, the 89-nt fragment was synthesized from the left probe annealing to the template, and the 60-nt fragment was synthesized from the reverse primer annealing to the right probe. When both the polymerase and the helicase were present, we detected 111-nt fragments labeled with either FAM or PET (Fig. 2b). Therefore, the helicase separated the newly synthesized double-stranded DNA and allowed the 89-nt and 60-nt fragments to anneal, which in turn allowed the polymerase to extend both strands. The 111-nt PET-labeled fragment serves as templates for subsequent amplification (Fig. 1). Of note, this fragment is complementary to the ligation product in MLPA, because the ligation product serves as the template for the reverse primer in MLPA (Fig. 1). Therefore, the generation of the 111-nt PET-labeled fragment strongly indicates that MLPA can occur without ligation.

We also assessed the fidelity of the reaction by substituting a single nucleotide in the template. The $3^{\prime}$ end of the left probe must match the template perfectly to be extended by the polymerase. We therefore generated a point mutation that mismatched the $3^{\prime}$ end of the left probe (Fig. 3). As expected, the left probe was barely extended in the mutant templates; however, small amounts of the 89- and 111-nt fragments were still detected (Fig. 3). Therefore, the mismatch between the $3^{\prime}$ end of the left probe and the template remarkably reduced but did not always prevent the extension of the left probe. Even slight extension of the left probe generates the 111-nt fragment, because the 60-nt PET-labeled fragment is generated irrespective of the template. The resulting 111-nt fragment is an ideal target for subsequent amplification; therefore, the high fidelity of the procedure could be lost after amplification.

\section{Ligation-independent probe amplification is functional}

To amplify the 111-nt PET-labeled fragment we used helicase-dependent amplification (HDA) rather than PCR because it has several advantages over PCR. ${ }^{11,12}$ HDA is similar to PCR except in the manner of denaturation. In HDA, a helicase separates double-stranded DNA at the temperature used for extension by the polymerase. Therefore, HDA essentially allows PCR to be accomplished isothermally without a thermocycler. Although HDA is difficult to optimize, it is a highly sensitive and rapid technique. ${ }^{13}$

For efficient amplification, we used a concentration of primers that was higher than that of the probes. The reactions were conducted at $65^{\circ} \mathrm{C}$ in the presence of both a thermostable helicase and a polymerase. Every product was analyzed chronologically with fragment analysis. The 111-nt fragment labeled with PET was amplified even without the forward primer (Fig. S1a, Supporting Information), and it was amplified efficiently when the forward primer was included (Fig. S1b, Supporting Information). The results demonstrate that the 


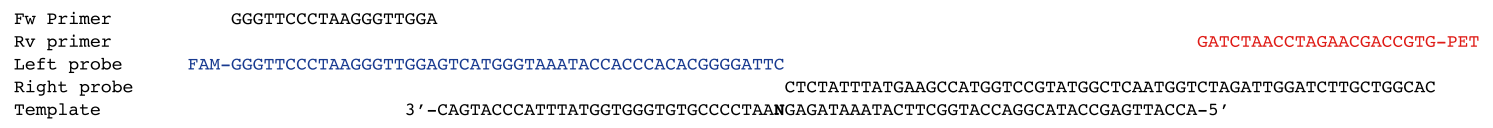

\section{a}
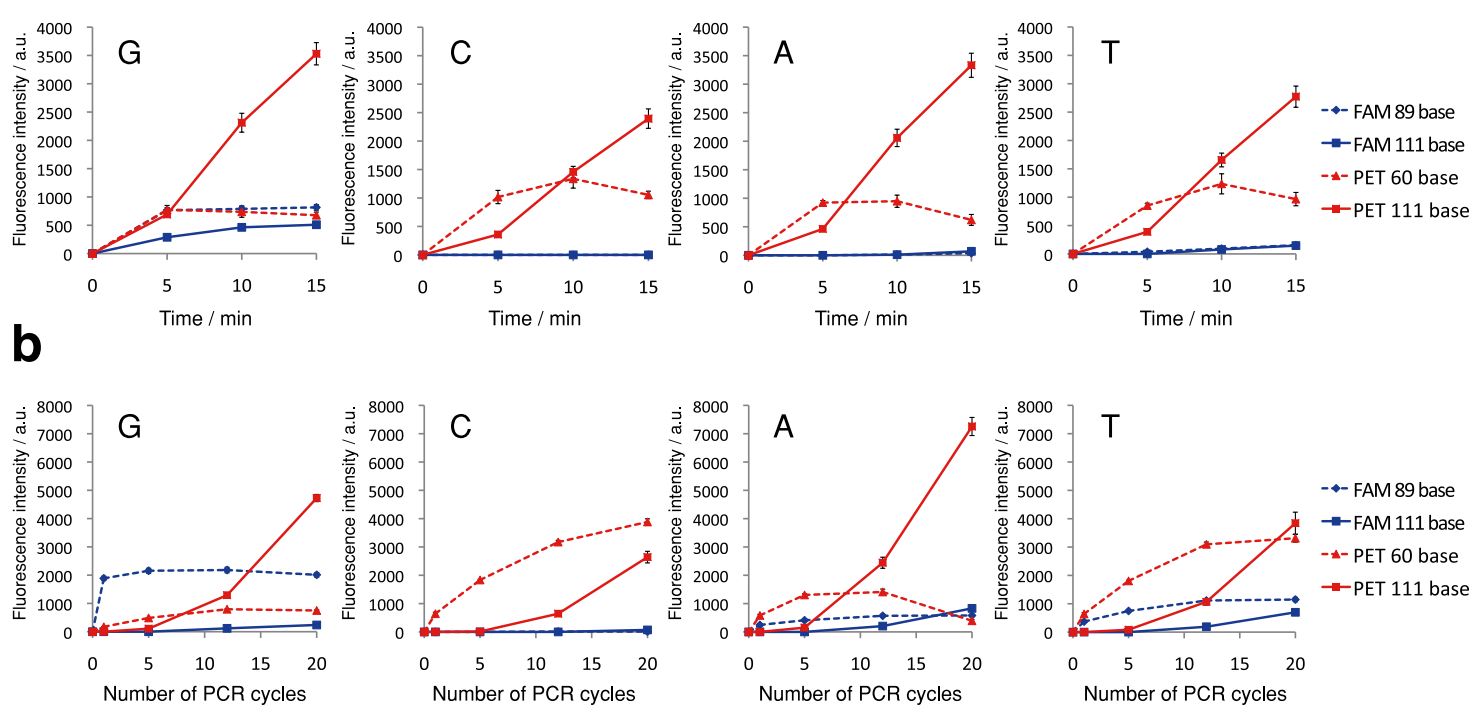

Fig. 4 Ligation-independent probe amplification is functional but not specific enough to distinguish a single nucleotide in the template. The reaction was performed using HDA (a) or PCR (b) in the presence of the forward primer and a template that differed by a single nucleotide (bold). The discriminating base is shown in each graph. Error bars indicate standard error of the mean (s.e.m). $n \geq 3$ (a) and $n=4$ (b). Representative electropherograms of (a) are shown in Fig. S2 (Supporting Information).

111-nt PET-labeled fragment was successfully amplified by HDA in a ligation-independent manner.

However, this fragment was also amplified from mutant templates (Fig. 4a and Supporting Information Fig. S2), which indicates that the left probe had extended slightly. Once the 111-nt PET-labeled fragment was generated in even small amounts, it was efficiently amplified by both primers. Therefore, efficiency is not always compatible with fidelity.

To confirm that MLPA occurs without ligation, we conducted the same reaction using PCR instead of HDA. The 111-nt PET-labeled fragment was clearly amplified (Fig. 4b), which provides direct evidence that MLPA is not dependent on ligation.

Ligation is insufficient to discriminate single-base changes in $M L P A$

We next carried out MLPA using the same oligonucleotides, but the $5^{\prime}$ end of the right probe was phosphorylated for ligation (Fig. 5a). We performed ligation using Taq ligase for $15 \mathrm{~min}$ followed by PCR using Taq polymerase in the same program described in the general MLPA protocol (MRC-Holland). Ligation was assessed by the presence of a 111-nt fragment labeled with FAM. Consistent with previous reports, ${ }^{14}$ the fidelity of thermostable ligase was considerably high (Fig. 5b). However, this fidelity was lost during PCR. Although mismatch ligation causes loss of fidelity in MLPA, the 111-nt fragment labeled with PET was amplified well, even when the ligation product was undetectable at the end of the ligation reaction (Fig. 5c and Supporting Information Fig. S3). The ligation reaction was almost completed at the beginning of PCR (data not shown). In addition, the amplification curves of the 111-nt PET-labeled fragment were similar to those obtained using ligation-independent probe amplification (Fig. 4). These results indicate that PCR amplified the 111-nt fragment labeled with PET in a ligation-independent manner. MLPA is reportedly accurate enough to distinguish single-base changes owing to ligation. However, our results show that high fidelity due to ligation can be lost during MLPA unless the PCR condition is optimized, and therefore MLPA is not always suitable for the analysis of single-nucleotide alterations. In fact, MLPA is mainly used for the detection of CNVs, not single-nucleotide changes.

\section{Discussion}

The present study draws attention to a basic understanding of MLPA, a process in which ligation is considered indispensable. The products of MLPA may be derived from a ligation-independent pathway, which should always be considered in the interpretation of MLPA results. We show that ligation does not always account for the fidelity of MLPA. However, MLPA works with great specificity for copy number analysis, perhaps because copy number analysis does not require accurate discrimination of single-base changes. MLPA is sufficiently specific as long as it is used for copy number analysis but not for single-nucleotide analysis because of the loss of the high fidelity of ligation. Our study suggests that the fidelity of MLPA depends on PCR conditions rather than ligation, because ligation-independent probe amplification using PCR was not specific enough to distinguish a single nucleotide in the template (Fig. 4b), but the optimization of PCR conditions such as annealing temperature or primer concentration ratio enabled us to discriminate a point mutation (data not shown). 
a

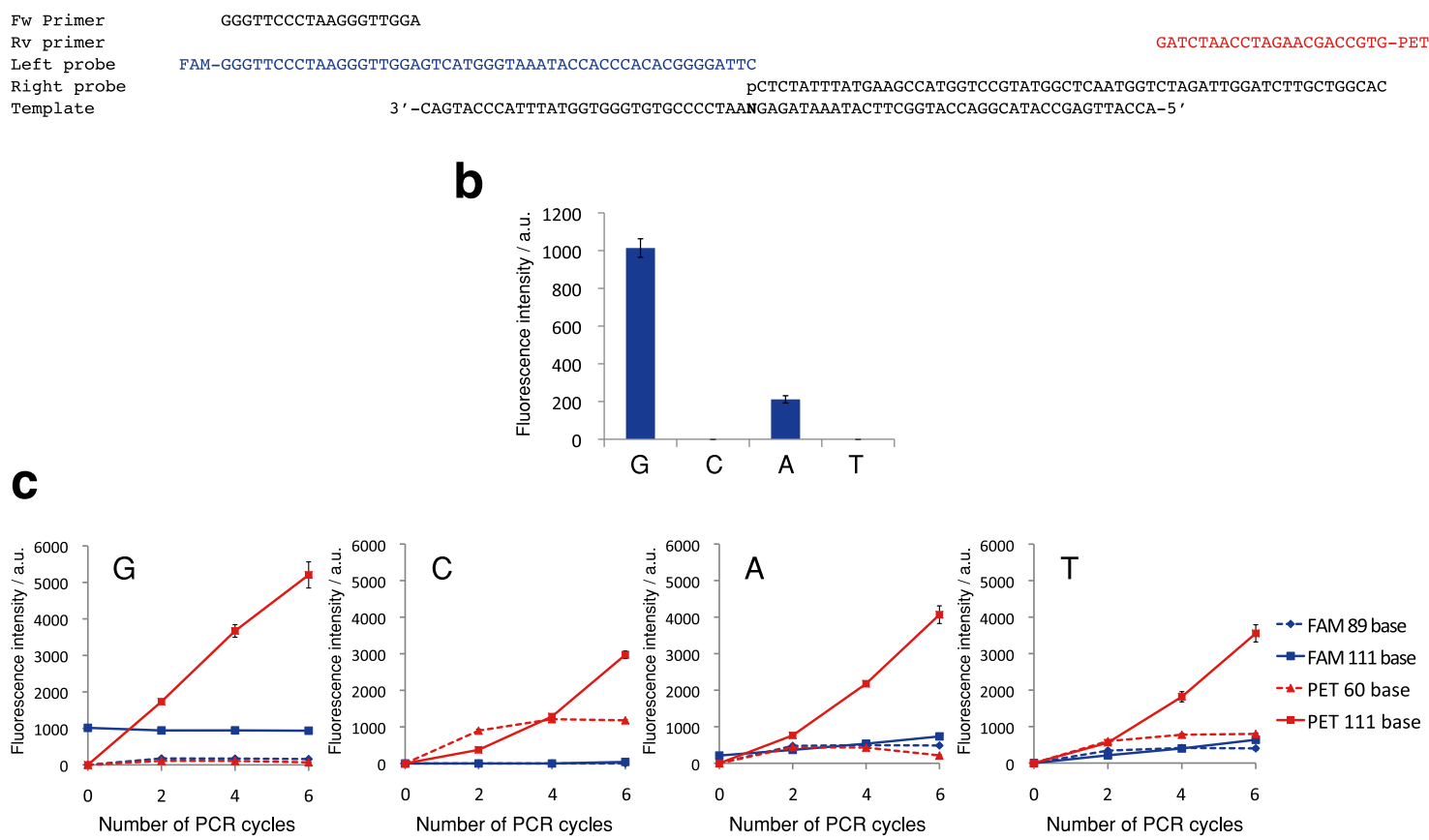

Fig. 5 The fidelity of MLPA does not rely on ligation. (a) Schematic representation of the oligonucleotides used in the reaction. MLPA was performed using a $5^{\prime}$-phosphorylated right probe and a template that differed by a single nucleotide (bold). (b) The ligation product was quantified by a 111$n t$ fragment labeled with FAM at the beginning of PCR $(0$ cycle). Error bars indicate s.e.m. $n=6$ (G and C) and $n=5$ (A and T). (c) The fidelity of ligation was lost during PCR. The discriminating base is shown in each graph. Error bars indicate s.e.m. $n=6(\mathrm{G}$ and C) and $n=5$ (A and T). Representative electropherograms are shown in Fig. S3 (Supporting Information).

\section{Acknowledgement}

This work was funded by Grants-in-Aid for Scientific Research (25870530) from the Ministry of Education, Culture, Sports, Science and Technology of Japan.

\section{Supporting Information}

Additional table and figures. This material is available free of charge on the Web at http://www.jsac.or.jp/analsci/.

\section{References}

1. J. P. Schouten, C. J. McElgunn, R. Waaijer, D. Zwijnenburg, F. Diepvens, and G. Pals, Nucleic Acids Res., 2002, 30, e57.

2. L. Feuk, A. R. Carson, and S. W. Scherer, Nat. Rev. Genet., 2006, 7, 85 .

3. B. S. Emanuel and S. C. Saitta, Nat. Rev. Genet., 2007, 8, 869.

4. S. H. Katsanis and N. Katsanis, Nat. Rev. Genet., 2013, 14 415.
5. L. Stuppia, I. Antonucci, G. Palka, and V. Gatta, Int. J. Mol. Sci., 2012, 13, 3245.

6. P. Kozlowski, A. J. Jasinska, and D. J. Kwiatkowski, Electrophoresis, 2008, 29, 4627.

7. P. Langerak, A. O. Nygren, J. P. Schouten, and H. Jacobs, Nucleic Acids Res., 2005, 33, e188.

8. C. Homig-Holzel and S. Savola, Diagn. Mol. Pathol., 2012, 21, 189.

9. I. Bergval, S. Sengstake, N. Brankova, V. Levterova, E. Abadia, N. Tadumaze, N. Bablishvili, M. Akhalaia, K. Tuin, A. Schuitema, S. Panaiotov, E. Bachiyska, T. Kantardjiev, R. de Zwaan, A. Schurch, D. van Soolingen, A. van't Hoog, F. Cobelens, R. Aspindzelashvili, C. Sola, P. Klatser, and R. Anthony, PloS One, 2012, 7, e43240.

10. I. L. Bergval, R. N. Vijzelaar, E. R. Dalla Costa, A. R. Schuitema, L. Oskam, A. L. Kritski, P. R. Klatser, and R. M. Anthony, J. Clin. Microbiol., 2008, 46, 689.

11. M. Vincent, Y. Xu, and H. Kong, EMBO Rep., 2004, 5, 795.

12. Y. J. Jeong, K. Park, and D. E. Kim, Cell. Mol. Life Sci., 2009, 66, 3325.

13. L. An, W. Tang, T. A. Ranalli, H. J. Kim, J. Wytiaz, and H. Kong, J. Biol. Chem., 2005, 280, 28952.

14. F. Barany, Proc. Natl. Acad. Sci. U. S. A., 1991, 88, 189. 